\title{
Hypoxia induces an increase in intestinal permeability and pulmonary arterial pressures in neonatal Holstein calves despite feeding the flavonoid rutin
}

\author{
Nicholas W. Shellenberger, ${ }^{1}$ Keleigh K. Collinsworth, ${ }^{1}$ Seenivasan Subbiah, ${ }^{2}$ David Klein, ${ }^{2}$ \\ and Joseph M. Neary ${ }^{3 *}$ (1) \\ ${ }^{1}$ Department of Animal and Food Sciences, College of Agricultural Sciences and Natural Resources, Texas Tech University, Lubbock 79409 \\ ${ }^{2}$ Department of Toxicology, The Institute of Environmental and Human Health, Texas Tech University, Lubbock 79409 \\ ${ }^{3}$ Department of Livestock Health and Welfare, Institute of Veterinary Science, University of Liverpool, Neston, CH64 7TE, UK
}

\section{ABSTRACT}

The purposes of this study were to determine whether the naturally occurring flavonoid quercetin, as its glucorhamnoside rutin, reduces intestinal permeability and susceptibility to hypoxia-induced pulmonary hypertension in neonatal Holstein calves. A $2 \times 2$ between-subjects factorial design was conducted using Holstein steers $(\mathrm{n}=16)$. Factors included oxygen level (simulated altitude of $4,572 \mathrm{~m}$ vs. $975 \mathrm{~m}$ ) and quercetin supplementation as its glucorhamnoside rutin ( $4 \mathrm{~g}$ of quercetin per day vs. $0 \mathrm{~g}$ per day). Two days after arrival (d 0 of study) the calves were blocked by body mass into treatment groups, and treatments were initiated. Pulmonary arterial pressure, echocardiography, and serum concentrations of orally administered lactulose $(0.45 \mathrm{~g} / \mathrm{kg})$ and mannitol $(0.15 \mathrm{~g} / \mathrm{kg})$ were measured on d 12, 13, and 14, respectively. Calves were euthanized on d 15 and pulmonary tissues collected for semiquantitative scoring of histological lesions. Data were analyzed using linear regression, generalized estimating equations, and 2-sample proportion tests. Hypoxia, but not rutin, was found to be associated with intestinal permeability. The lactulose-mannitol ratio was $0.54 \pm 0.13$ (standard error) in hypoxic calves and $0.02 \pm 0.13$ in normoxic controls. Hypoxia increased mean pulmonary arterial pressure. Calves fed rutin under hypoxic conditions tended to have a lower mean pulmonary arterial pressure $(59 \pm 7 \mathrm{mmHg})$ than control calves $(80 \pm 7 \mathrm{mmHg}$ ) but similar pressures under normoxic conditions. Paradoxically, however, a greater proportion of calves fed rutin had histological evidence of pulmonary arteriolar medial hypertrophy

Received July 18, 2019.

Accepted November 13, 2019.

*Corresponding author: J.Neary@liverpool.ac.uk and adventitial hyperplasia than did controls. In conclusion, the findings of this study indicate that hypoxia increased intestinal permeability in neonatal calves. The flavonoid quercetin, as its glucorhamnoside rutin, had no protective effect on intestinal permeability, and, although it tended to reduce the severity of hypoxiainduced pulmonary hypertension, a greater proportion of calves fed rutin had histological lesions consistent with pulmonary arteriolar remodeling.

Key words: leaky gut, bacteremia, liver abscess, passive transfer

\section{INTRODUCTION}

Increased gastrointestinal permeability can be deleterious to health. In cattle, gastrointestinal permeability is most commonly problematic in neonatal calves and in cattle fed high-starch finishing diets before slaughter. In neonates, the translocation of gastrointestinal bacteria into the bloodstream may lead to septicemia, meningitis, and other focal infections, particularly in association with failure of passive transfer (Fecteau et al., 2009). Antimicrobials are the most important therapeutic modality for treatment of calf septicemia (Fecteau et al., 2009). In feedlot cattle, low ruminal $\mathrm{pH}$ associated with high-starch diets is thought to predispose to the passage of pyogenic bacteria through the damaged mucosa and into the blood (Nagaraja and Lechtenberg, 2007). As a prophylactic, $71.2 \pm 4.4 \%$ (SE) of all feedlots feed the broad-spectrum macrolide antibiotic tylosin (USDA, 2011). Antimicrobial-free interventions to improve gut barrier function could, therefore, have broad benefits across the cattle industry.

Evidence from rodent and animal studies indicates that the naturally occurring dietary flavonoid quercetin has 2 major health benefits: reducing gastrointestinal permeability (Li et al., 2016; Bhutto et al., 2018) and reducing susceptibility to pulmonary hypertension (Duarte et al., 2001; Perez-Vizcaino et al., 2009; Panchal et al., 2011; Morales-Cano et al., 2014), a potentially 
lethal physiological state that is becoming increasingly problematic in cattle (Neary et al., 2016a; Krafsur et al., 2019). Holstein-Friesian and Jersey breeds of dairy cattle are known to be susceptible to hypoxia-induced pulmonary hypertension, and subsequent death loss can be problematic in regions over $1,500 \mathrm{~m}$ in altitude (Malherbe et al., 2012) and secondary to respiratory compromise (Holt and Callan, 2007). The hypoxic effects of respiratory disease may be particularly deleterious in calves because lung function does not become optimal until approximately 1 yr of age (Lekeux et al., 1984; Neary et al., 2014). By preventing the production of inflammatory cytokines such as TNF $\alpha$ and enzymes such as cyclooxygenase, lipoxygenase, and poly(ADPribose) polymerase-1 (Geraets et al., 2007; Li et al., 2016), quercetin may counter the inflammatory effects of hypoxia (Eltzschig and Carmeliet, 2011). Quercetin also acts as a gastrointestinal protectant through a stabilizing effect on leukocytes (Middleton et al., 2006) and intestinal tight-junction proteins (Amasheh et al., 2008; Suzuki and Hara, 2009). We hypothesized that dietary supplementation with the naturally occurring flavonoid quercetin, provided as its glucorhamnoside rutin, will decrease mucosal permeability to the nondigestible, nonmetabolizable sugars mannitol and lactulose, and reduce the susceptibility of calves to hypoxia-induced pulmonary arterial hypertension.

\section{MATERIALS AND METHODS}

\section{Study Site}

Day-old Holstein bull calves were obtained from a large commercial dairy farm within 40 miles of the Texas Tech University research farm (New Deal, TX). A total of 16 calves were studied, in 2 blocks of 8 calves. The first block was collected on February 19, 2018, and the second was collected on March 20, 2018. Calves were fed $4 \mathrm{~L}$ of colostrum within $6 \mathrm{~h}$ of birth, according to calf management protocol. Serum total proteins were measured at $2 \mathrm{~d}$ of age to assess adequacy of passive immunoglobulin transfer.

Calves were group-housed $\left(\mathrm{n}=4 ; 1.5 \mathrm{~m}^{2}\right.$ per calf $)$ in pens (Agri-Plastics, Grassie, ON, Canada) on raised perforated flooring in 1 of 2 chambers $\left(41 \mathrm{~m}^{3}\right)$. Temperature $\left(15 \pm 2^{\circ} \mathrm{C}\right)$, light ( $12 \mathrm{~h}$ light, $12 \mathrm{~h}$ dark), and oxygen levels (Altitude Training Systems, Arndell Park, Australia) were controlled within the chambers. Hypoxic oxygen levels remained within $\pm 0.5 \%$ of the target simulated altitude throughout the study. Normoxic controls were exposed to atmospheric air. Each chamber was equipped with a dehumidifier (4-gallon dehumidifier, model HME020030N; HomeLabs, New
York, NY) fitted with a continuous drain hose. Temperature and humidity ranges were checked daily using an indoor digital monitor (TP50 Digital Hygrometer; ThermoPro, Atlanta, GA).

The calves were weighed $1 \mathrm{~d}$ after arrival; the heaviest 4 calves were assigned to one chamber, and the 4 lightest were assigned to the other. Calves were stratified into dietary treatment groups according to failure of passive transfer status $(<55 \mathrm{~g} / \mathrm{L}$ of serum total protein; Weaver et al., 2000). Within each stratum, calves were randomly assigned to dietary treatment groups so that, within each chamber, 2 calves were fed the dietary additive rutin and 2 served as negative controls. The first block of calves was housed under normoxic conditions (altitude $975 \mathrm{~m}$ ) and the second block under hypoxic conditions (simulated altitude 4,572 m). Simultaneous normoxic and hypoxic conditions could not be achieved due to equipment constraints.

\section{Feed and Husbandry}

Starting on the morning after arrival, calves were individually fed $3 \mathrm{~L}$ of milk replacer (High-Energy Nurse Chow; Purina, St. Louis, MO), $22 \%$ protein, and $12 \%$ fat at $15 \%$ milk solids, twice per day using standard, commercially available plastic calf bottles and nipples. Milk replacer was prepared in a large bucket using warm water (approximately $43^{\circ} \mathrm{C}$ ) and a balloon whisk. Texturized calf starter (Purina Ampli-Calf Starter 22), $22 \%$ protein and $2.5 \%$ fat, and water were available ad libitum throughout the study. Feed was checked daily for spoilage and replaced every other day.

Treatment exposure began $2 \mathrm{~d}$ after arrival (d 0 of the study). Calves received $4 \mathrm{~g}$ of rutin trihydrate $(97 \%$, Alfa Aesar, Heysham, UK) per day, divided into $2 \mathrm{~g}$ per meal. Rutin was weighed in disposable plastic boats to a precision of $0.01 \mathrm{~g}$ (Ohaus SPX1202, Ohaus Corporation, Parsippany, NJ). The rutin was then poured into the bottle containing milk replacer, which was capped and inverted several times. Calves were hand-fed using designated bottles to prevent control calves from consuming rutin. Bottles were hand-washed and dried after each feeding.

\section{Health Monitoring}

Calves were assessed daily for signs of respiratory distress, tachypnea, and scours by a veterinarian throughout the study, and rectal temperatures measured on alternate days. Breathing rate and effort were assessed from a distance before the lungs and trachea were auscultated. Fecal scoring was performed using 4 categories: $0=$ normal, $1=$ semi-formed, $2=$ loose 
but not watery, $3=$ sufficiently watery to easily pass through slatted floor. Blood-gas analyses were periodically performed on auricular arterial blood solely to assess hypoxic status (results not shown). Electrolytes (Re-sorb, Zoetis, Kalamazoo, MI) were given to calves with fecal score $\geq 3$ in place of the morning feed. No antibiotics were used in the study.

\section{Intestinal Permeability Evaluation}

Calves were weighed on d 14, immediately before oral administration of $0.15 \mathrm{~g} / \mathrm{kg}$ of D-Mannitol (Thermo Fisher Scientific, Bridgewater, NJ) and $0.45 \mathrm{~g} / \mathrm{kg}$ of lactulose (99\%, Alfa Aesar). Both substances were weighed using disposable plastic boats to a precision of $0.01 \mathrm{~g}$ (SPX1202, Ohaus Corporation) before mixing with $0.5 \mathrm{~L}$ of water (approximately $43^{\circ} \mathrm{C}$ ). The mannitol and lactulose solution was then fed using an esophageal feeder to minimize the amount of solution lost during the feeding process. A blood sample was collected from the jugular vein $4 \mathrm{~h}$ later. Measurements of serum mannitol and lactulose concentrations obtained every 2 $\mathrm{h}$ until $8 \mathrm{~h}$ post-feeding revealed peak serum concentrations at $4 \mathrm{~h}$ after feeding (Gonyeau et al., 2017). The serum samples were stored at $-20^{\circ} \mathrm{C}$. Calves were fed their regular morning milk replacer $2 \mathrm{~h}$ after the mannitol and lactulose challenge.

To extract serum proteins in preparation for liquid chromatography-tandem mass spectrometry (LCMS/MS) analysis, samples were thawed at room temperature and $100 \mu \mathrm{L}$ of homogenized serum sample transferred to a microcentrifuge tube $(2 \mathrm{~mL}$, Eppendorf, Hauppauge, NY). Next, $300 \mu \mathrm{L}$ of solvent mixture containing acetonitrile (LC/MS Optima grade, Thermo Fisher Scientific) and water (LC/MS Optima grade, Thermo Fisher Scientific; 80:20 vol/vol) was transferred to the tube. After $5 \mathrm{~min}$ at room temperature, the tube, now containing serum and solvent solution, was vortexed for $30 \mathrm{~s}$ before centrifugation at 21,130 $\times g$ for $10 \min$ (5424 R, Eppendorf). The supernatant was transferred into a syringeless filter vial (PTFE, $0.45 \mu \mathrm{m}$, GE Healthcare UK Ltd., Chalfont St. Giles, UK) for LC-MS/MS analysis. Standards were prepared using $10 \mathrm{mg}$ of the standard in $10 \mathrm{~mL}$ (mannitol) or $15 \mathrm{~mL}$ (lactulose) of solvent (50:50 vol/ vol methanol: water). From these, serial dilutions of $1,5,10,25,50$, and $100 \mu \mathrm{g} / \mathrm{mL}$ in a mixture of acetonitrile and water (80/20 vol $/ \mathrm{vol})$ were prepared.

Ultra high-pressure liquid chromatography-mass spectrometry was performed on a liquid chromatograph with triple-staged quadrupole mass spectrometer (Ultimate 3000 TSQ Endura, Thermo Fisher Scientific).
Serum extract $(5 \mu \mathrm{L})$ was injected into an RP-Amide column (Accentis RP-Amide, $5 \mu \mathrm{m}, 50 \times 2.1 \mathrm{~mm}$; Sigma-Aldrich, St. Louis, MO). The column and autosampler tray temperatures were $45^{\circ} \mathrm{C}$ and $10^{\circ} \mathrm{C}$, respectively. Mobile phases A and B consisted of $0.1 \%$ formic acid (LC/MS Optima grade, Thermo Fisher Scientific) in water and $0.1 \%$ formic acid in acetonitrile, respectively. The flow rate was $0.4 \mathrm{~mL} / \mathrm{min}$.

\section{Pulmonary Arterial Pressure Measurement}

Calves were manually restrained using a lamb trimming stand for pulmonary arterial pressure measurement on d 12. The neck was clipped and cleaned with chlorhexidine solution. A scalpel blade was used to create a full-thickness stab incision through an intradermal lidocaine bleb placed in the jugular groove. A 7-French peel-away introducer (IS-07AS, Vascor Medical Corporation, Tarpon Springs, FL) was placed in the jugular vein before the insertion of a 110-cm 7-French polyurethane modified J-tip wedge pressure catheter (172-110P, Vascor Medical Corporation). A pressure transducer (TranStar DPT, Smiths Medical ASD Inc., Dublin, $\mathrm{OH}$ ) connected the catheter to the data acquisition system (IX-TA-220, iWorx Systems Inc., Dover, $\mathrm{NH})$. The transducer was taped horizontally to a retort ring that was attached to a 1-m laboratory stand. The height of the retort ring was adjusted so that the transducer was dorsal to the point of the elbow, the approximate location of the pulmonary arterial trunk. The pressure waveforms were recorded and analyzed offline (LabScribe3, iWorx Systems Inc.). Pressures were analyzed at the end of expiration. The position of the catheter tip was determined by monitoring the change in the pressure waveform as the catheter tip was advanced through the right atrium, right ventricle, and finally into the pulmonary artery.

\section{Echocardiography}

Right parasternal short-axis views of the left ventricle, midway between the papillary muscles and mitral valve, were obtained on d 13 using a portable ultrasound machine (Vivid i, General Electric, Boston, $\mathrm{MA}$ ) and 1.5-4.0 $\mathrm{MHz}$ sector probe (3Sc-RS, General Electric). End-systolic and end-diastolic left ventricular eccentricity index values $\left(\mathbf{L}_{1}, \mathbf{L}_{2}\right)$ were calculated from the septal-posterolateral $\left(\mathrm{L}_{2}\right)$ and anterior-inferior cavity $\left(\mathrm{L}_{1}\right)$ dimensions. The $\mathrm{L}_{1}$ dimension ran parallel to the septum at the widest aspect and level with the chordae tendinae. The $\mathrm{L}_{2}$ dimension bisected the septum and was perpendicular to $\mathrm{L}_{1}$, as shown in Figure 1 . 


\section{Histology}

Calves were euthanized on d 15 (pentobarbital sodium $85 \mathrm{mg} / \mathrm{kg}$, i.v.) and exsanguinated immediately postmortem. A gross examination of all major organs was performed, and tissue sections approximately $1-\mathrm{cm}$ thick obtained from the dorsal aspect of the left diaphragmatic lung lobe and preserved in neutral buffered formalin $(10 \% ; 10: 1$ formalin to tissue volume). The left lung lobes were gravity-perfused with formalin through the main bronchi for 10 min before tissue sample collection so that the lung parenchyma was preserved in its expanded state. Tissues were trimmed and paraffinized approximately 1 wk later. Slides containing sections 4 $\mu \mathrm{m}$ thick were stained with hematoxylin and eosin. The severity of lesions were scored semiquantitatively $(0=$ no lesion; $+1=$ mild; $+2=$ moderate $;+3=$ severe) as performed elsewhere (Malherbe et al., 2012; Neary et al., 2013). Muscular pulmonary arteries below 500- $\mu \mathrm{m}$ diameter were scored, due to their selective involvement in brisket disease. Image analysis software was used for measurements and scale bar creation (Rueden et al., 2017).

\section{Lung Weight}

The right lung lobes of all calves were weighed and placed on a metal wire drying rack in one of the chambers previously used to house the calves. The lungs were kept in the chamber at $90^{\circ} \mathrm{C}$, with the dehumidifiers continuously running, until there was less than a 1-g change in the lung weight over consecutive days.

\section{Statistical Analyses}

Statistical analyses were performed using commercially available software (Stata 15.1, StataCorp, College Station, TX). Data were descriptively analyzed graphically and numerically to assess data distribution and the presence of outliers. Backward stepwise linear regression was performed with the full model containing both factor variables and their interaction. Clustering by chamber was included as a fixed effect. Results are presented as marginal means \pm standard error of the mean unless otherwise stated. Likelihood ratio tests were used to test the overall statistical significance of a variable where an interaction was statistically significant or where an interaction was hypothesized. An $\alpha$-value of 0.05 was used throughout the analyses. Post-estimation procedures were performed to assess model fit. Repeated rectal temperature measurements were assessed using generalized estimating equations and an exchangeable correlation structure. Two-tailed, 2-sample proportions tests were undertaken to evaluate whether the proportion of calves with at least mild histological lesions differed between treatments.

\section{RESULTS}

\section{Descriptive}

Calves weighed $40.8 \pm 4.5 \mathrm{~kg}$ (mean $\pm \mathrm{SD}$ ) on arrival at the university farm and $44.1 \pm 3.3 \mathrm{~kg}$ on the day of euthanasia. We found no effect of treatment on calf body mass at the start or end of the study $(P>$ $0.52)$. Four calves (2 per block) had failure of passive
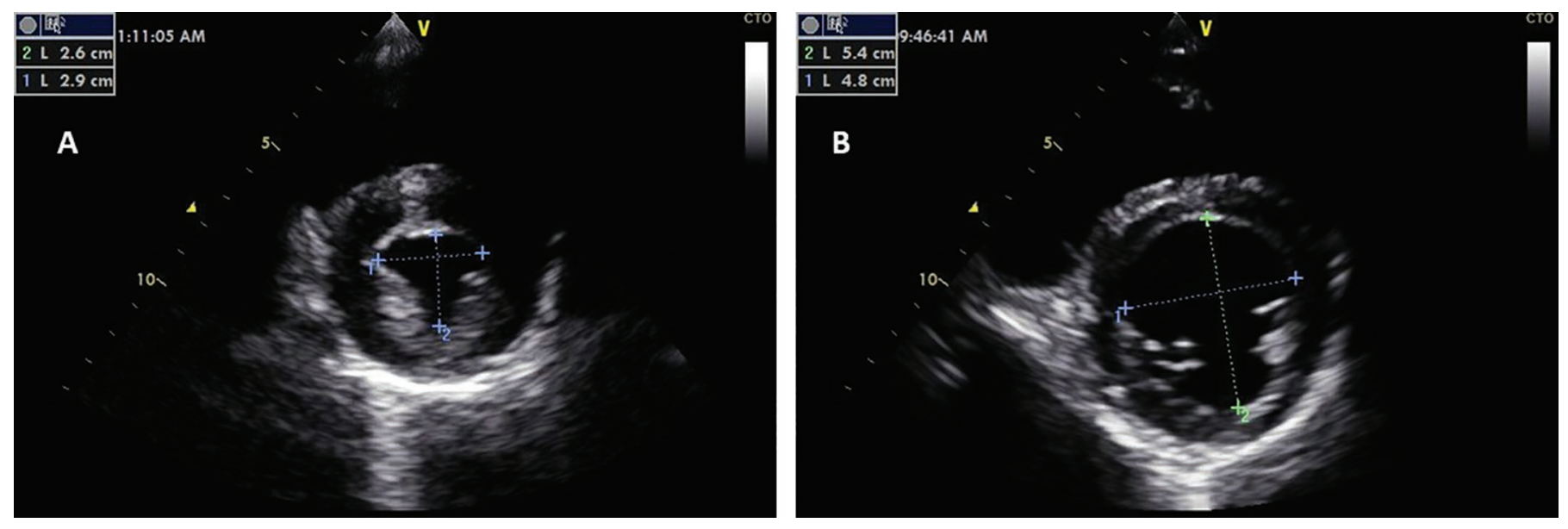

Figure 1. Right parasternal short-axis views of the left ventricle, midway between the papillary muscles and mitral valve, demonstrating measurement of systolic (A) and diastolic (B) eccentricity indexes $\left(\mathrm{L}_{1} / \mathrm{L}_{2}\right)$ in 2 calves housed under hypoxic conditions and not fed rutin. Eccentricity did not differ among treatment groups, and no evidence of septal flattening occurred in association with pulmonary hypertension. 
transfer. These calves were equally distributed among treatments. Four calves were administered oral electrolytes for the treatment of scours.

\section{Gastrointestinal Permeability to Lactulose and Mannitol}

Hypoxia, but not the feeding of rutin, was associated with intestinal permeability. Serum lactulose was greater in hypoxic $(0.60 \pm 0.13)$ than normoxic calves $(0.13 \pm 0.13 ; P=0.03)$ but we found no difference between calves fed $(0.40 \pm 0.13)$ and not fed $(0.33 \pm$ $0.13)$ rutin $(P=0.75)$. Serum mannitol was greater in hypoxic $(1.16 \pm 0.25)$ than normoxic calves $(0.45$ $\pm 0.25 ; P=0.03)$ but no difference occurred between calves fed $(0.93 \pm 0.25)$ and not fed $(0.68 \pm 0.25)$ rutin $(P=0.50)$. The lactulose-to-mannitol ratio was greater in hypoxic $(0.54 \pm 0.13)$ than normoxic calves $(0.02$ $\pm 0.13 ; P=0.02)$, but no difference occurred between calves fed $(0.38 \pm 0.13)$ and not fed $(0.18 \pm 0.13) \mathrm{ru}-$ tin $(P=0.31)$. We found no interaction between the dietary additive rutin and hypoxia on blood serum concentrations of lactulose $(P=0.09)$, mannitol $(P=$ $0.75)$, and the ratio of lactulose to mannitol $(P=0.15)$.

\section{Effect of Rutin and Hypoxia on Pulmonary Pressure}

Hypoxia $(P<0.001)$ and the dietary additive rutin $(P=0.07)$ were associated with mean pulmonary arterial pressure (mPAP). The interaction between hypoxia and rutin was in the direction hypothesized $(P=0.13)$. Calves fed rutin under hypoxic conditions had a lower mPAP $(59 \pm 7 \mathrm{mmHg})$ than control calves $(80 \pm 7 \mathrm{mmHg})$. Under normoxic conditions, calves fed rutin had similar mPAP $(30 \pm 7 \mathrm{mmHg})$ to control calves $(28 \pm 7 \mathrm{mmHg})$.

\section{Echocardiography}

Analysis revealed no effect of hypoxia $(P=0.81)$ or rutin $(P=0.83)$ on left ventricular end-diastolic eccentricity index $(1.21 \pm 0.05$; Figure 1). Nor did an effect of hypoxia $(P=0.82)$ or rutin $(P=0.93)$ occur on left ventricular end-systolic eccentricity index $(1.26 \pm$ 0.09). We found no pairwise correlation between mPAP and left ventricular end-diastolic eccentricity index $(P$ $=0.57)$ and between mPAP and left ventricular endsystolic eccentricity index $(P=0.66)$.

\section{Rectal Temperature}

Hypoxia $(P=0.42)$ and the dietary additive rutin $(P$ $=0.91)$ were not associated with calf rectal temperature. On average, rectal temperature increased by 0.13 $\pm 0.01^{\circ} \mathrm{C}$ per week of the study $(P=0.03)$ and was 38.4 $\pm 0.2^{\circ} \mathrm{C}$ on $\mathrm{d} 1$.

\section{Lung Weight}

Rutin had no effect on the proportion of lung mass attributable to water $(P=0.67)$. However, the percentage water content was marginally lower in calves exposed to hypoxia $(80.3 \pm 0.3 \%)$ than normoxic controls $(81.6 \pm 0.3 \% ; P=0.017)$.

\section{Histology}

Grossly, the postmortem findings were unremarkable, with no evidence of disease present. Five hypoxic calves and 3 normoxic calves had at least mild lesions of pulmonary arteriolar hypertrophy $(P=0.31$; Table 1$)$. Six calves fed rutin and 2 control calves had at least mild lesions of pulmonary arteriolar medial hypertrophy $(P$ $=0.045)$. Five hypoxic calves and 4 normoxic calves had at least mild pulmonary arteriolar adventitial hyperplasia $(P=0.61$; Figure 2$)$. Seven calves fed rutin and 2 control calves had at least mild pulmonary arteriolar adventitial hyperplasia $(P=0.01)$.

\section{DISCUSSION}

This study found that the naturally occurring flavonoid quercetin, as its glucorhamnoside rutin, had no effect on intestinal permeability in neonatal Holstein

Table 1. Frequency table of pulmonary arteriolar hypertrophy and pulmonary arteriolar adventitial hyperplasia according to dietary rutin and environmental hypoxia

\begin{tabular}{|c|c|c|c|c|c|}
\hline Lesion & Severity & \multicolumn{2}{|c|}{ Normoxic $(\mathrm{n}=8)$} & \multicolumn{2}{|c|}{ Hypoxic $(\mathrm{n}=8)$} \\
\hline \multirow[t]{2}{*}{ Pulmonary arteriolar medial hypertrophy } & None & 3 & 2 & 3 & 0 \\
\hline & Moderate & 1 & 0 & 1 & 1 \\
\hline \multirow[t]{2}{*}{ Pulmonary arteriolar adventitial hyperplasia } & None & 3 & 1 & 3 & 0 \\
\hline & Mild & 0 & 3 & 1 & 4 \\
\hline
\end{tabular}


calves. Although rutin reduced mPAP in calves housed under hypoxic conditions, it was deleteriously associated with pulmonary arteriolar remodeling. Hypoxia, however, significantly increased both mPAP and intestinal permeability.

Hypoxia-induced pulmonary hypertension in bovine calves has been well documented (Stenmark et al., 1987; Neary et al., 2013, 2016b), but hypoxia-induced gastrointestinal permeability has not. However, numerous studies in rodent models demonstrate the deleterious effect of hypoxia on intestinal permeability (Fisher et al., 2013; Novosad et al., 2013). Unlike rodents and other mammals, cattle are reported to be particularly susceptible to hypoxemia due to their small lung volume relative to body mass (Jensen et al., 1976; Veit and Farrell, 1978; Kainer and Will, 1981). Immature lung function (Lekeux et al., 1984; Neary et al., 2014), respiratory compromise, and hypobaric hypoxia (Gulick et al., 2016) could all feasibly contribute toward a hypoxia-induced increase in intestinal permeability with deleterious consequences on animal health. Hypobaric hypoxia, even at the modest altitude of 975 $\mathrm{m}$, appears to have predisposed the normoxic control calves in our study to pulmonary vascular remodeling, as no difference occurred in the proportion of calves with at least mild arterial lesions between those housed under hypoxic conditions versus normoxic controls. We suspect that these results may have been confounded somewhat by activity level, as the hypoxic calves were more sedentary throughout the study. Hypoxic calves had a lower proportion of lung weight attributable to water. This may have been attributable to greater hypoxia-induced remodeling within the pulmonary parenchyma and vasculature of calves housed under hypoxic conditions.

Contrary to our hypothesis, the antioxidant rutin was not effective at reducing intestinal permeability or pulmonary arterial pressure. This may be attributable to a variety of causes, from poor bioavailability to rapid metabolism and excretion. Poor aqueous solubility and bioavailability may be improved through use of encapsulation techniques, which, in a study of rabbits, improved rutin bioavailability 4-fold (Ramaswamy et al., 2017). Without technologies such as encapsulation, the application of insoluble additives such as rutin and quercetin to milk replacer would be impractical, as the additives would deposit out of the solution unless the feeding vessel is frequently agitated to keep the additive suspended in solution.

Alternatively, our findings may be attributable to pro-oxidative activities, which may occur at high doses of anti-oxidants or in the presence of metal ions. Determining a suitable dosage was problematic because studies evaluating the bioavailability of quercetin and rutin in ruminants are limited (Berger et al., 2012; Maciej et al., 2015). At high concentrations $(>50 \mu M)$, quercetin has been shown to exhibit pro-oxidative activity in vitro (Robaszkiewicz et al., 2007; De Marchi et al., 2009; Bouayed and Bohn, 2010). At lower concentrations, however, quercetin and its rhamnoglucoside rutin have a wide range of biological activities and pharmacological effects, such as antihypertensive, vasoprotective, and cardioprotective activities (Panchal et al., 2011; Yeh et al., 2014; Monori-Kiss et al., 2017). One study
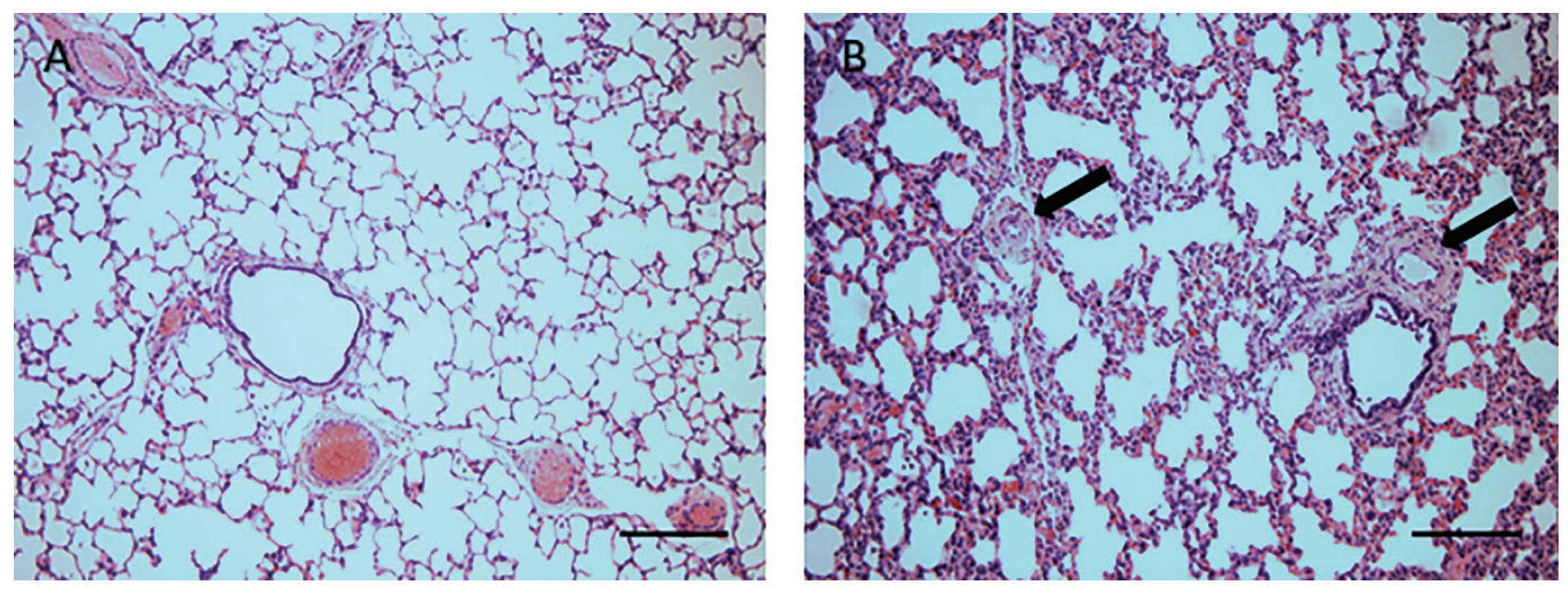

Figure 2. Pulmonary lesions of the left diaphragmatic lung lobe after gravity perfusion with $10 \%$ formalin in a calf housed at a simulated altitude of 4,572 m, with no evidence of pulmonary arteriolar medial hypertrophy or adventitial hyperplasia (A), and a calf housed at an altitude of $975 \mathrm{~m}$, showing evidence (arrows) of moderate pulmonary arteriolar medial hypertrophy and adventitial hyperplasia (B). Neither calf consumed rutin. Hematoxylin and eosin staining. Scale bar $=200 \mu \mathrm{m} .100 \times$ magnification. 
found that quercetin lowered pulmonary arterial pressure and reversed vascular remodeling in male Wistar rats (Morales-Cano et al., 2014). In our study, rutin was associated with lower mean pulmonary arterial pressure under hypoxic conditions, but, paradoxically, a greater proportion of calves fed rutin had histological lesions consistent with pulmonary arteriolar remodeling. The reason for this is unclear, but we suspect that rutin had pro-oxidative effects. Unfortunately, however, we cannot confirm this, because in vivo antioxidant activity was not measured. Perhaps of greater interest is the presence of pulmonary arteriolar lesions in normoxic controls that are consistent with pulmonary hypertension. Mean pulmonary arterial pressures in humans and other mammalian species are less than $25 \mathrm{mmHg}$ at rest; consequently, the presence of pulmonary vascular lesions and relatively high pressures in normoxic neonatal bovine calves at the moderate altitude of 975 $\mathrm{m}$ warrants closer attention, as some have suggested an etiological link to respiratory disease susceptibility (Veit and Farrell, 1978).

The potential confounding of hypoxic exposure due to separation into separate cohorts or blocks is a limitation of this study. Unfortunately, this was unavoidable due to equipment restraints, which prevented simultaneous study of normoxic and hypoxic calves. Potential confounding was minimized by obtaining both blocks of calves from the same farm and within a 4 -wk period. We also tested for adequacy of passive transfer and found no differences between blocks. This could, however, have been problematic because gastrointestinal pathogens could have confounded the effect of hypoxia on intestinal permeability. Interestingly, neonatal calves that subsequently developed scours were reported to have greater intestinal permeability than those calves that did not (Araujo et al., 2015).

In conclusion, the purposes of this study were to determine whether the naturally occurring flavonoid quercetin, as its glucorhamnoside rutin, has favorable effects on reducing gastrointestinal permeability and reducing susceptibility to hypoxia-induced pulmonary hypertension in neonatal Holstein calves. Although the study found that rutin had no effect on intestinal permeability in neonatal Holstein calves, we also found that hypoxia significantly increases permeability. Drug delivery techniques for rutin and other hydrophobic substances require further study for successful incorporation into the preweaned diet.

\section{ACKNOWLEDGMENTS}

Funding for this study was provided by the Texas Cattle Feeders Association (Amarillo, TX). The authors have no conflicts of interest to declare.

\section{REFERENCES}

Amasheh, M., S. Schlichter, S. Amasheh, J. Mankertz, M. Zeitz, M. Fromm, and J. D. Schulzke. 2008. Quercetin enhances epithelial barrier function and increases claudin-4 expression in Caco-2 cells. J. Nutr. 138:1067-1073. https://doi.org/10.1093/jn/138.6.1067.

Araujo, G., C. Yunta, M. Terré, A. Mereu, I. Ipharraguerre, and A. Bach. 2015. Intestinal permeability and incidence of diarrhea in newborn calves. J. Dairy Sci. 98:7309-7317. https://doi.org/10 $.3168 /$ jds.2015-9666.

Berger, L. M., S. Wein, R. Blank, C. C. Metges, and S. Wolffram. 2012. Bioavailability of the flavonol quercetin in cows after intraruminal application of quercetin aglycone and rutin. J. Dairy Sci 95:5047-5055. https://doi.org/10.3168/jds.2012-5439.

Bhutto, Z. A., F. He, M. Zloh, J. Yang, J. Huang, T. Guo, and L. Wang. 2018. Use of quercetin in animal feed: Effects on the P-gp expression and pharmacokinetics of orally administrated enrofloxacin in chicken. Sci. Rep. 8:4400. https://doi.org/10.1038/s41598 -018-22354-1.

Bouayed, J., and T. Bohn. 2010. Exogenous antioxidants-Doubleedged swords in cellular redox state: Health beneficial effects at physiologic doses versus deleterious effects at high doses. Oxid Med. Cell. Longev. 3:228-237. https://doi.org/10.4161/oxim.3.4 .12858 .

De Marchi, U., L. Biasutto, S. Garbisa, A. Toninello, and M. Zoratti. 2009. Quercetin can act either as an inhibitor or an inducer of the mitochondrial permeability transition pore: A demonstration of the ambivalent redox character of polyphenols. Biochim. Biophys. Acta 1787:1425-1432. https://doi.org/10.1016/j.bbabio.2009.06 .002 .

Duarte, J., R. Pérez-Palencia, F. Vargas, M. Angeles Ocete, F. PérezVizcaino, A. Zarzuelo, and J. Tamargo. 2001. Antihypertensive effects of the flavonoid quercetin in spontaneously hypertensive rats. Br. J. Pharmacol. 133:117-124. https://doi.org/10.1038/sj .bjp.0704064.

Eltzschig, H. K., and P. Carmeliet. 2011. Hypoxia and inflammation. N. Engl. J. Med. 364:656-665. https://doi.org/10.1056/ NEJMra0910283.

Fecteau, G., B. P. Smith, and L. W. George. 2009. Septicemia and meningitis in the newborn calf. Vet. Clin. North Am. Food Anim. Pract. 25:195-208. https://doi.org/10.1016/j.cvfa.2008.10.004.

Fisher, E. M., M. Khan, R. Salisbury, and P. Kuppusamy. 2013. Noninvasive monitoring of small intestinal oxygen in a rat model of chronic mesenteric ischemia. Cell Biochem. Biophys. 67:451-459. https://doi.org/10.1007/s12013-013-9611-y.

Geraets, L., H. J. J. Moonen, K. Brauers, E. F. M. Wouters, A. Bast, and G. J. Hageman. 2007. Dietary flavones and flavonoles are inhibitors of poly(ADP-ribose)polymerase-1 in pulmonary epithelial cells. J. Nutr. 137:2190-2195. https://doi.org/10.1093/jn/137.10 .2190 .

Gonyeau, K., S. Subbiah, D. Klein, D. Church, and J. Neary. 2017. Development of pulmonary arterial hypertension and diffuse alveolar damage in 2-month old Holstein dairy calves following an acute episode of bloody scours. F1000 Res. 6:1826. https://doi.org/10 $.12688 /$ f1000research.12717.1.

Gulick, A. K., F. B. Garry, T. N. Holt, K. Retallick-Trennepohl, R. M. Enns, M. G. Thomas, and J. M. Neary. 2016. Angus calves born and raised at high altitude adapt to hypobaric hypoxia by increasing alveolar ventilation rate but not hematocrit. J. Anim. Sci. 94:4167-4171. https://doi.org/10.2527/jas.2016-0718.

Holt, T. N., and R. J. Callan. 2007. Pulmonary arterial pressure testing for high mountain disease in cattle. Vet. Clin. North Am. Food Anim. Pract. 23:575-596. https://doi.org/10.1016/j.cvfa.2007.08 .001 .

Jensen, R., R. E. Pierson, P. M. Braddy, D. A. Saari, A. Benitez, D. P. Horton, L. H. Lauerman, A. E. McChesney, A. F. Alexander, and D. W. Will. 1976. Brisket disease in yearling feedlot cattle. J. Am. Vet. Med. Assoc. 169:515-517.

Kainer, R. A., and D. A. Will. 1981. Morphophysiologic bases for the predisposition of the bovine lung to bronchial pneumonia. Prog. Clin. Biol. Res. 59B:311-317. 
Krafsur, G. M., J. M. Neary, F. Garry, T. Holt, D. H. Gould, G. L. Mason, M. G. Thomas, R. M. Enns, R. M. Tuder, M. P. Heaton, R. D. Brown, and K. R. Stenmark. 2019. Cardiopulmonary remodeling in fattened beef cattle: A naturally occurring large animal model of obesity-associated pulmonary hypertension with left heart disease. Pulm. Circ. 9:2045894018796804. https://doi.org/10 $.1177 / 2045894018796804$.

Lekeux, P., R. Hajer, and H. J. Breukink. 1984. Effect of somatic growth on pulmonary function values in healthy Freisian cattle. Am. J. Vet. Res. 45:2003-2007.

Li, Y., J. Yao, C. Han, J. Yang, M. Chaudhry, S. Wang, H. Liu, and Y. Yin. 2016. Quercetin, inflammation and immunity. Nutrients 8:167. https://doi.org/10.3390/nu8030167.

Maciej, J., C. T. Schäff, E. Kanitz, A. Tuchscherer, R. M. Bruckmaier, S. Wolffram, and H. M. Hammon. 2015. Bioavailability of the flavonol quercetin in neonatal calves after oral administration of quercetin aglycone or rutin. J. Dairy Sci. 98:3906-3917. https:/ /doi.org/10.3168/jds.2015-9361.

Malherbe, C. R., J. Marquard, D. E. Legg, K. M. Cammack, and D. O'Toole. 2012. Right ventricular hypertrophy with heart failure in Holstein heifers at elevation of 1,600 meters. J. Vet. Diagn. Invest. 24:867-877. https://doi.org/10.1177/1040638712453580.

Middleton, E., C. Kandaswami, and C. T. Theoharides. 2006. The effect of plant flavonoids on mammalian cells: Implications for inflammation, heart disease, and cancer. Pharmacol. Rev. 52:673751 .

Monori-Kiss, A., F. Kiss, J. M. Restifo, E. Monos, and G. L. Nadasy. 2017. Chronic administration of quercetin induces biomechanical and pharmacological remodeling in the rat coronary arteries. Physiol. Res. 66:591-599.

Morales-Cano, D., C. Menendez, E. Moreno, J. Moral-Sanz, B. Barreira, P. Galindo, R. Pandolfi, R. Jimenez, L. Moreno, A. Cogolludo, J. Duarte, and F. Perez-Vizcaino. 2014. The flavonoid quercetin reverses pulmonary hypertension in rats. PLoS One 9:e114492. https://doi.org/10.1371/journal.pone.0114492.

Nagaraja, T.G., and K.F. Lechtenberg. 2007. Liver abscesses in feedlot cattle. Vet. Clin. North Am. Food Anim. Pr. 23:351-369. https:// doi.org/10.1016/j.cvfa.2007.05.002.

Neary, J. M., C. W. Booker, B. K. Wildman, and P. S. Morley. 2016a. Right-sided congestive heart failure in North American feedlot cattle. J. Vet. Intern. Med. 30:326-334. https://doi.org/10.1111/ jvim.13789.

Neary, J. M., R. D. Brown, T. N. Holt, K. R. Stenmark, R. M. Enns, M. G. Thomas, and F. B. Garry. 2016b. Static and dynamic components of right ventricular afterload are negatively associated with calf survival at high altitude. J. Anim. Sci. 94:4172-4178. https://doi.org/10.2527/jas.2016-0652.

Neary, J. M., F. B. Garry, and S. M. Raabis. 2014. Age-related changes in arterial blood-gas variables in Holstein calves at moderate altitude. Open Access Anim. Physiol. 6:13-20. https://doi.org/10 .2147/OAAP.S60976.

Neary, J. M., D. H. Gould, F. B. Garry, A. P. Knight, D. A. Dargatz, and T. N. Holt. 2013. An investigation into beef calf mortality on five high-altitude ranches that selected sires with low pulmonary arterial pressures for over 20 years. J. Vet. Diagn. Invest. 25:210218. https://doi.org/10.1177/1040638713478608.
Novosad, V. L., J. L. Richards, N. A. Phillips, M. A. King, and T. L. Clanton. 2013. Regional susceptibility to stress-induced intestinal injury in the mouse. Am. J. Physiol. Gastrointest. Liver Physiol. 305:G418-G426. https://doi.org/10.1152/ajpgi.00166.2013.

Panchal, S. K., H. Poudyal, T. V. Arumugam, and L. Brown. 2011. Rutin attenuates metabolic changes, nonalcoholic steatohepatitis, and cardiovascular remodeling in high-carbohydrate, high-fat dietfed rats. J. Nutr. 141:1062-1069. https://doi.org/10.3945/jn.111 .137877 .

Perez-Vizcaino, F., J. Duarte, R. Jimenez, C. Santos-Buelga, and A. Osuna. 2009. Antihypertensive effects of the flavonoid quercetin. Pharmacol. Rep. 61:67-75. https://doi.org/10.1016/s1734 $-1140(09) 70008-8$.

Ramaswamy, S., L. P. Dwarampudi, M. Kadiyala, G. Kuppuswamy, K. Veera Venkata Satyanarayana Reddy, C. K. A. Kumar, and M. Paranjothy. 2017. Formulation and characterization of chitosan encapsulated phytoconstituents of curcumin and rutin nanoparticles. Int. J. Biol. Macromol. 104:1807-1812. https://doi.org/10.1016/j .ijbiomac.2017.06.112.

Robaszkiewicz, A.. A. Balcerczyk, and G. Bartosz. 2007. Antioxidative and prooxidative effects of quercetin on A549 cells. Cell Biol. Int. 31:1245-1250. https://doi.org/10.1016/j.cellbi.2007.04.009.

Rueden, C. T., J. Schindelin, M. C. Hiner, B. E. DeZonia, A. E. Walter, E. T. Arena, and K. W. Eliceiri. 2017. ImageJ2: ImageJ for the next generation of scientific image data. BMC Bioinformatics 18:529. https://doi.org/10.1186/s12859-017-1934-z.

Stenmark, K. R., J. Fasules, D. M. Hyde, N. F. Voelkel, J. Henson, A. Tucker, H. Wilson, and J. T. Reeves. 1987. Severe pulmonary hypertension and arterial adventitial changes in newborn calves at 4,300 m. J. Appl. Physiol. 62:821-830. https://doi.org/10.1152/ jappl.1987.62.2.821.

Suzuki, T., and H. Hara. 2009. Quercetin enhances intestinal barrier function through the assembly of zonnula occludens-2, occludin and claudin- 1 and the expression of claudin- 4 in Caco-2 cells. J. Nutr. 139:965-974. https://doi.org/10.3945/jn.108.100867.

USDA. 2011. Feedlot 2011, Part IV: Health and Health Management on U.S. Feedlots with a Capacity of 1,000 or More Head. USDAAPHIS-VS-CEAH-NAHMS, Fort Collins, CO.

Veit, H. P., and R. L. Farrell. 1978. Anatomy and physiology of bovine respiratory system relating to pulmonary disease. Cornell Vet. 68:555-581.

Weaver, D. M., J. W. Tyler, D. C. VanMetre, D. E. Hostetler, and G. M. Barrington. 2000. Passive transfer of colostral immunoglobulins in calves. J. Vet. Intern. Med. 14:569-577. https://doi.org/10 .1111/j.1939-1676.2000.tb02278.x.

Yeh, C. H., J. J. Yang, M. L. Yang, Y. C. Li, and Y. H. Kuan. 2014. Rutin decreases lipopolysaccharide-induced acute lung injury via inhibition of oxidative stress and the MAPK-NF- $\kappa \mathrm{B}$ pathway. Free Radic. Biol. Med. 69:249-257. https://doi.org/10.1016/j .freeradbiomed.2014.01.028.

\section{ORCIDS}

Joseph M. Neary ৫i https://orcid.org/0000-0001-8438-2234 